\title{
Risky Behaviors and Associated Factors among the Elderly in Rural Vietnam
}

\author{
Dinh Le Mai ${ }^{1,{ }^{*}}$, Nguyen Van Huy ${ }^{2}$, Nguyen Van Thanh ${ }^{3}$, and Henning Staar ${ }^{4}$ \\ ${ }^{1}$ Department of Molecular Biology, 108 Military Central Hospital, Vietnam \\ ${ }^{2}$ Department of Health Management and Organization, Institute for Preventive Medicine and Public Health, Hanoi Medical University, Vietnam \\ 3 Institute for Preventive Medicine and Public Health, Hanoi Medical University, Vietnam \\ ${ }^{4}$ Business Psychology, BiTS - Business and Information Technology School GmbH, University of Applied Sciences, Germany
}

*Corresponding author: Dinh Le Mai, Department of Molecular Biology, 108 Military Central Hospital, Tran Hung Dao Str., Hai Ba Trung Dist., Hanoi, Vietnam, Tel: +84.32595488; E-mail: maisu10193@gmail.com

Received Date: June 10, 2017; Accepted Date: July 18, 2017; Published Date: July 22, 2017

Copyright: (c) 2017 Mai DL, et al. This is an open-access article distributed under the terms of the Creative Commons Attribution License, which permits unrestricted use, distribution, and reproduction in any medium, provided the original author and source are credited.

\begin{abstract}
Background: The number of older people in Vietnam has increased substantially for recent years and leading to more aging health problems. The purpose of this study was to assess risky behaviors and their associated factors among the elderly in rural Vietnam.

Design: In a cross-sectional study using quantitative methods, 600 elderly ( $\geq 60$ years old) living in a rural district in Northern Vietnam in 2013 were approached for data collection. Logistic regression was undertaken to identify factors associated with each of the risky behaviors, including using alcohol, smoking, and physical inactivity.

Results: The frequency of drinking, smoking and physical inactivity among the rural elderly was quite prevalent, $25.17 \%, 22.17 \%$ and $60 \%$, respectively. The multivariate regression analysis revealed that alcohol use was most strongly predicted by gender (male) and age (60-79 years old). Further, smoking behavior was significantly more prevalent among those who were male, younger age, with low social participation, low social support and high social cohesion. In terms of physical inactivity, being female, married, having a caregiver, with low social participation, low social cohesion and high social support were most likely to predict inadequate exercises among the rural older people.
\end{abstract}

Conclusions: Social efforts are important to create social conditions (social participation, social cohesion and social support) that facilitate healthy behaviors among the seniors. The results can be utilized to inform appropriate intervention programs in other similar settings of developing countries.

Keywords: Risky behavior(s); Alcohol use; Smoking; Physical inactivity; Elderly/old people; Rural; Vietnam

\section{Introduction}

The world's population is expanding and aging. The number of people aged 60 and above is 841 million in 2013, which is four times estimated higher than the 202 million in 1950 [1]. In Vietnam, the number of older adults will reach 10 percent of the total population in 2017, which means that the Vietnamese population will enter the socalled "aging phase" from the year 2017 on [2]. This increasing trend could lead to health problems, such as chronic diseases, associated with aging and is supposed to have profound implications for the healthcare system as well as the economic growth for coming years [3]. According to WHO, behavior and exposure to health risks such as smoking, alcohol consumption and physical inactivity can have long-term health implications and thus, negatively influence health in older age [4]. Therefore, understanding these risky behaviors and some linked factors among older people is essential for developing effective prevention programs to improve health and reduce disease burden of the elderly.

Alcohol is defined by WHO as "a psychoactive substance with dependence-producing properties" and drinking or consumption of alcohol have causes a great amount of social and health consequences in many communities around the world [5]. Although the elderly are more susceptible to the adverse effects of alcohol than their younger counterparts due to their generally poorer health condition and negative alcohol interactions with medications [6-8], alcohol use among older people is still prevalent [7,9]. In Sweden, all age-groups decreased their average alcohol consumption between 2002 and 2007 except for people aged 65 years or over whose consumption increased [10]. A study in the Netherlands revealed that the frequency of alcohol use was highest in the group of 65 years and above, with $30.6-32.7 \%$ of men and 20.2-22.0\% of women drinking 6-7 times per week [9]. According to a research by Siegfried Weyerer, half of Germans aged 75 and older continued drinking alcohol throughout their old age. The study also showed that alcohol consumption was significantly associated with male gender, younger age, higher level of education, not living alone and not being depressed [11]. Another study in Northern Thailand among older people (50 years and above) pointed out that lower education, low income and moral barriers were related with daily alcohol use [12]. In addition, other studies indicated some socio-demographic factors associated with alcohol use of the elderly including being male $[9,13,14]$, smoking $[9,15]$, being socially isolated $[16,17]$ and being single or separated $[15,18]$. Although some previous studies have explored alcohol consumption among the Vietnamese 
elderly $[19,20]$, the factors associated with it have not been systematically examined.

Smoking is considered any active actions when "a chemical toxicosis which is able to causes detrimental effects either of acute or chronic type on different structures of the body" [21]. In terms of tobacco use, it is one of the avoidable leading causes of disability and death among the elderly [22,23]. Older adults who smoke have a higher risk of dementia and have double the mortality rate as compared to older non-smokers [24-27]. A meta-analysis of 48 studies found that the overall prevalence of tobacco use among the elderly was $13 \%$ in both gender (22\% male and $8 \%$ female) [28]. In 2010, a study conducted in 17 European countries found that the overall smoking prevalence among older adults ( $\geq 65$ years old) was $11.5 \%$ ( $15.3 \%$ among men and $8.6 \%$ among woman). This study also indicated positive associations with current smoking status and a lower education level as well as low national implementations of tobacco control activities [29]. According to a study in Canada, being female, increased age, having a higher income, living with someone else and being active in social participation were negatively associated with smoking status of the elderly [30]. Another study conducted in Iran found that smoking was significantly higher among men, the elderly living alone and the elderly who had heart diseases [31]. Regarding to the data of Global Adult Tobacco Survey in 2010, Vietnam is one of the 15 top consumers of tobacco in the world with over 15 million smokers. Whereas a large body of research conducted about smoking behavior focuses on adolescents and adults [32-36], little information is known about the tobacco use rate and related factors among the elderly in Vietnam.

WHO stated that "Physical activity is defined as any bodily movement produced by skeletal muscles that require energy expenditure and physical inactivity is lacking physical activity" [37]. Physical inactivity has been also identified as the fourth leading risk factor for global mortality (3.2 million deaths each year) [37]. It is estimated to be the main cause for approximately $21-25 \%$ of breast colon cancers, $27 \%$ of diabetes and $30 \%$ of ischemic heart disease [38] and consequently affect quality of life among older adults [39]. According to WHO recommendations, older people should undertake a minimum of 30 minutes of moderate intensive physical activity at least 5 days a week to promote their health. However, physical inactivity remains a common phenomenon among many groups of populations, including the elderly. For example, a study conducted in Shanghai, China pointed out that $83.3 \%$ of the elderly living alone did not have adequate physical activity per week [40]. The study by Adelle et al. revealed that $62 \%$ ( $66.3 \%$ of the women and $54.7 \%$ of the men) of rural Brazilian older adults had not engaged in specific physical activity in the prior three months. There are many factors affecting this behavior. For example, the study on rural Brazilian older people found that physical inactivity was positively associated with being female, increased age, lower education, lower income and lack of participation in social activities [41]. Other study in Australia detected an inverse relationship between physical inactivity and sex (male), younger age, and rural residence [42]. However, data about physical activity or exercise among the elderly in Vietnam is of scarcity and is usually conducted as a part of a foreign-funded research with small Vietnamese sample $[43,44]$. Therefore, examining physical inactivity levels and influential factors among Vietnamese older people will provide crucial evidence for policy makers to design interventions to improve their health.

Rural elderly population is considered a vulnerable group [45] and they are facing many challenges, especially when their government does not provide proper support [46]. In terms of accessing medical services, there are many barriers for the elderly in rural areas such as transportation difficulties, lack of health care supply, limited quality health care, social isolation and financial constraints [47-49]. In Vietnam, there are $72.9 \%$ of the elderly living in rural areas with disadvantaged living conditions [50]. Besides, $70 \%$ of rural older adults in Vietnam suffer from non-communicable diseases, especially cardiovascular diseases, diabetes, kidney diseases and cancer [51,52]. However, this disease burden of rural older can decrease by cutting down on health-risk behaviors, including using alcohol, smoking and physical inactivity.

Given a relatively limited profile on this topic, the current study was conducted to examine risky behaviors and associated factors among the elderly in rural Vietnam. The study findings should inform suitable health behavior change programs for Vietnamese rural older.

\section{Methods}

\section{Design}

This is a cross-sectional study using face-to-face interviews assisted with structured quantitative questionnaire.

\section{Study site and time}

This survey was carried out in two rural communes of Kien Xuong district in Thai Binh province, northern Vietnam. Kien Xuong is an agricultural district with a large area of $200 \mathrm{~km} 2$ and a population of 274,318 people, including 34,898 senior citizens [53]. We chose these two communes as the study site because they could represent a typical rural community in Vietnam such as income sources mainly from agriculture, being quite far from urban area and has an increasing number of the seniors $[53,54]$. The overall research duration was from January 2013 to August 2013.

\section{Sample}

The older adults who aged 60 years or above, living in the selected areas for at least 5 years, being available at the survey time, and of normal mentality and voluntary participation were regarded as suitable study subjects. We used the threshold of 60 years of age as elderly based on the current context of Vietnam. To be specific, according to Vietnamese elderly law in 2009 stipulating that any citizen who is more than or equal to 60 years old is considered elderly [55]. The sample size was calculated using the formula estimating a population proportion with specified relative precision:

$$
n=Z_{1-\alpha / 2}^{2} \frac{p(1-p)}{(p \varepsilon)^{2}}
$$

where $\mathrm{n}$ is sample size $Z_{1-\alpha / 2}^{2}$ is the reliability coefficient; take $\alpha=0.05$ with the $95 \%$ confidence interval, then $Z_{1-\alpha / 2}^{2}=1.96$. $\mathrm{p}$ is the percentage of older people who have the risky behaviors from previous studies. In a previous study in Thailand in 2015, the percentage of old people (aged 60 and above) who were current drinkers was $63.5 \%$. In another study in Iran in 2014, the percentage of current smokers among the elderly was $39.3 \%$. In a previous study in Shanghai, China in 2015, the proportion of older people who did not have adequate physical activity was $83.3 \%$. Therefore, we take $\mathrm{p}=0.393$ to get the maximum sample. $\varepsilon$ is relative precision, the relative 
precision in our study was set at 0.1 . Using the values, a sample of 600 participants was calculated.

This study sample was recruited using a systematic random sampling approach, including two phases. Phase 1 included a sample of two communes in Kien Xuong district from Thai Binh province. In phase $2 \mathrm{k}$-interval in each commune was identified (average of $\mathrm{k}$ interval=4) from the lists of older people living in the two selected communes provided by the People's Committee. The number of participants in each commune was selected using systematic random sampling until a required sample size was met. Both sampling and interviews were conducted by lecturers and researchers from the Faculty of Public Health, Thai Binh University of Medicine and Pharmacy, Vietnam. They were well experienced with the previous community-based surveys. In total, 600 old people from the selected communes were recruited and interviewed.

\section{Measurement}

Dependent variables (main indicators): There are three main indicators, alcohol drinking, cigarette smoking and physical inactivity. Drinking behavior was determined by the yes/no question "Did you drink alcohol last month?". Smoking behavior was assessed by the yes/no question "Did you smoke cigarette last month?". Physical inactivity behavior was identified by the question "Were you engaged in any kind of exercise activities or sport last month?" The respondents who answered in the affirmative were regarded as being physically active. The others who denied the question were regarded as being physically inactive.

Independent variables (predictors): Demographic information was collected including age groups (60-79 and $\geq 80$ years old); marital status (single, divorced, widowed, and married); religion (no religion, Christianity and Buddhism); educational level (illiteracy, not completing primary level, not completing secondary level, secondary level or above); living circumstance (living with spouse, children, friends, relatives or living alone); caregiver (yes, no); socio-economic status (low, medium and high) based on the subjective assessment of the respondents.

Social indicators included social participation, social cohesion and social support. Social participation was measured with eight yes/no items of eight social groups (retirement clubs, women's associations, veterans' associations, Communist Party cells, sport clubs, street population groups, religious groups and senior groups (Cronbach's alpha =0.67). The number of organizations that an individual participates corresponding to the total score, with a higher score indicating a higher level of social participation.

Social cohesion was measured by five yes/no questions as follows: "Do you talk to your neighbors or the local government on the issues of your community?", "Do you join with other people in your residential area to solve problems in your community?", "In general, are people living in your residential area harmony with each other?", "Do you feel yourself connected and be a part of your resident area?", "Do you think that those who live in your residential area want to take advantage of your strengths?"; with Cronbach's alpha $=0.62$. Each "Yes" response was considered one point and a higher aggregate score indicated a higher social cohesion of the participant.

Social support, defined as receiving any emotional or material support from individuals and social organizations, was assessed by six dichotomous items: Respondents were asked if they received social support from any family members, from friends (not neighbors), from neighbors, from government support services, from the residential community leaders, from charities/non-governmental organizations/ religious organizations; with Cronbach's alpha $=0.60$. Each support is equal 1 point and a higher total point representing a higher social support.

\section{Data collection}

Prior to the main survey, the field researchers were trained to become familiar with the key contents of the research, survey methods, sampling methods, selecting study subjects, recording the responses of study subjects. The survey questionnaire was then pretested on a sample of 50 old people. The pilot result showed that the questionnaire was technically feasible for the main survey. The official survey was finally conducted if respondents met the criteria of inclusion. Those subjects who were not available at the interview time were set another appointment. Each interview lasted about 30-45 minutes. The interviews were conducted between June and August 2013. During the survey, the principal investigator was always present in the field to supervise data collection.

\section{Data analysis}

Data were cleaned by checking missing data before being entered into database using EPIDATA version 3.1 software. The data continued to be cleaned for outlier and illogical data and converted for analysis by STATA 12.0 Software. Descriptive statistics were used to present the participants' characteristics. Inferential statistics were performed using multivariate logistic regression with the effect significant at the $p<.05$ level. Three predictive models were examined separately to find out factors associated with drinking, smoking and physical inactivity behavior among the elderly. The final models were decided based on the multivariate analysis indices and the factors that significantly contributed to the model.

\section{Research ethics}

The research was approved by the Scientific and Education Panel from the Institute for Preventive Medicine and Public Health, Hanoi Medical University. Before the main survey started, the subjects were clearly provided with goals and contents of the study. Respondents were informed that their participation was completely voluntary and they could leave the study anytime if they did not want to continue. All collected information were kept confidential and only served for the research purpose.

\section{Results}

\section{Sample characteristics}

The characteristics of respondents are described in table 1. A total of 600 older people (53.67\% female and $46.33 \%$ male) in rural Thai Binh province were sampled.

The majority of participants were 60-79 years old (84.67\%), married (74.5\%), non-religious (75.5\%) and having a caregiver to care for themselves (90.17\%). As reported by the respondents, a caregiver can be their children, friends, neighbors or family relatives. The mean score $( \pm S D$ ) of social participation, social cohesion and social support was $1.15 \pm 1.08,3.42 \pm 1.28$ and $2.45 \pm 1.40$, respectively. Regard to education levels, $40.33 \%$ of the respondents completed primary level, followed by secondary level $(31.33 \%)$ and high school level or above 
Citation: Mai DL, Huy NV, Thanh NV, Staar H (2017) Risky Behaviors and Associated Factors among the Elderly in Rural Vietnam. J Aging Sci 5: 182. doi:10.4172/2329-8847.1000182

Page 4 of 9

(28.33\%). Most of the subjects reported having medium socioeconomic status $(60.83 \%)$.

\begin{tabular}{|c|c|c|}
\hline Variables $(n=600)$ & Frequency $(\%)$ & Mean ( \pm SD) \\
\hline \multicolumn{3}{|l|}{ Demographic information } \\
\hline \multicolumn{3}{|l|}{ Gender } \\
\hline Female & $322(53.67)$ & \\
\hline Male & $278(46.33)$ & \\
\hline \multicolumn{3}{|l|}{ Age } \\
\hline $60-79$ years old & $508(84.67)$ & \\
\hline$\geq 80$ years old & $92(15.33)$ & \\
\hline \multicolumn{3}{|l|}{ Marital status } \\
\hline Single & $39(6.5)$ & \\
\hline Married & $447(74.5)$ & \\
\hline Divorced/ Widow & $114(19)$ & \\
\hline \multicolumn{3}{|l|}{ Religion } \\
\hline No religion & $453(75.5)$ & \\
\hline Religion (Christianity/Buddhism) & $147(24.5)$ & \\
\hline \multicolumn{3}{|l|}{ Educational level } \\
\hline Primary level & $242(40.33)$ & \\
\hline Secondary level & $188(31.33)$ & \\
\hline High school level or above & $170(28.33)$ & \\
\hline \multicolumn{3}{|l|}{ Living circumstance } \\
\hline Living with spouse & $432(72)$ & \\
\hline Living with children & $303(50.5)$ & \\
\hline Living with grandchildren & $163(27.17)$ & \\
\hline Living alone & $38(6.33)$ & \\
\hline Caregiver & $541(90.17)$ & \\
\hline \multicolumn{3}{|l|}{ Socio-economic status } \\
\hline Low & $167(27.83)$ & \\
\hline Medium & $365(60.83)$ & \\
\hline High & $68(11.33)$ & \\
\hline \multicolumn{3}{|l|}{ Social capital } \\
\hline Social participation score (range $0-8$ ) & & $1.15 \pm 1.08$ \\
\hline Social cohesion score (range $0-5$ ) & & $3.42 \pm 1.28$ \\
\hline Social support score (range $0-6$ ) & & $2.45 \pm 1.40$ \\
\hline
\end{tabular}

Table 1: The characteristics of respondents. 
Citation: Mai DL, Huy NV, Thanh NV, Staar H (2017) Risky Behaviors and Associated Factors among the Elderly in Rural Vietnam. J Aging Sci 5:

Page 5 of 9

\section{Pattern of risky behaviors among the sample}

Table 2 represents the prevalence of risky behaviors among the Vietnamese rural elderly. The most common risky behavior among the elderly was physical inactivity (60\%).

\begin{tabular}{|l|l|}
\hline Risky behaviors $(\mathbf{n}=\mathbf{6 0 0})$ & $\mathbf{n}(\mathbf{\%})$ \\
\hline Drinking & $151(25.17)$ \\
\hline Smoking & $133(22.17)$ \\
\hline Physical inactivity & $360(60)$ \\
\hline Smoking and drinking & $94(15.67)$ \\
\hline Smoking and physical inactivity & $67(11.17)$ \\
\hline Drinking and physical inactivity & $73(12.17)$ \\
\hline Smoking and drinking and physical inactivity & $48(8)$ \\
\hline
\end{tabular}

Table 2: Prevalence of risky behaviors among the elderly in rural Vietnam.

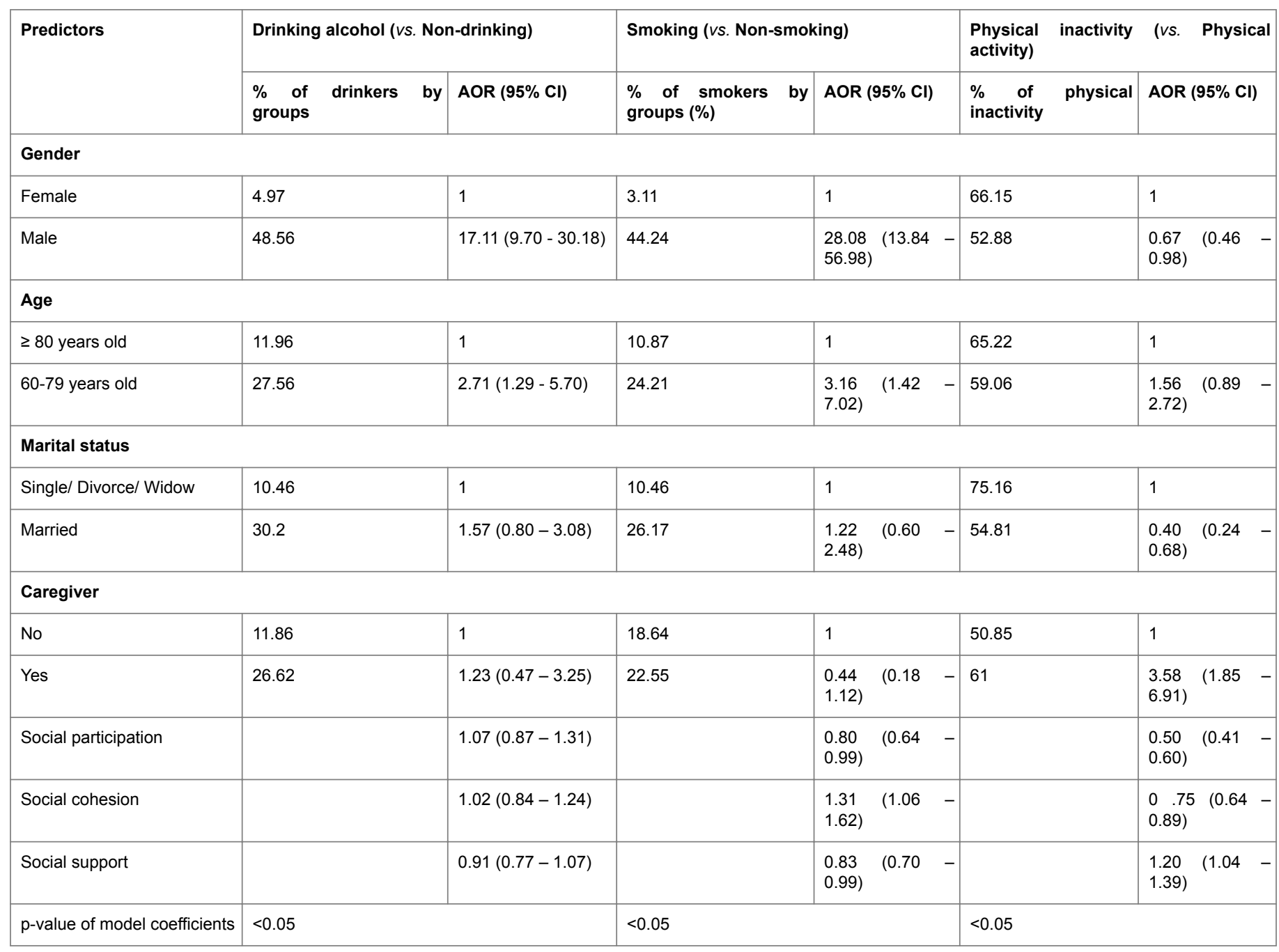

Of the total 600 subjects, the overall drinking rate was one-fourth $(25.17 \%)$ and the prevalence of current smoking was $22.17 \%$. The proportion of respondents who reported having both smoking and drinking, both drinking and physical inactivity, both smoking and physical inactivity was $15.67 \%, 12.17 \%$ and $11.17 \%$, respectively. Finally, there were 48 older people $(8 \%)$ those who revealed all of the three risky behaviors.

\section{Factors associated with risky behaviors (drinking, smoking and physical inactivity) among the elderly in rural Vietnam}

Table 3 summarizes the results of the multiple logistic regressions of the factors associated with drinking, smoking and physical inactivity among the elderly. 
Page 6 of 9

\begin{tabular}{|l|l|l|l|}
\hline $\begin{array}{l}\text { p-value }[x 2(\mathrm{df}) \text { of Hosmer } \\
\text { and Lemeshow] }\end{array}$ & NS & NS & NS \\
\hline Nagelkerke's R2 & $26.93 \%$ & $29.44 \%$ & $15.25 \%$ \\
\hline
\end{tabular}

Table 3: Multivariate logistic regression of factors associated with risky behaviors among the Vietnamese rural elderly. Note: CI: Confidence Interval; AOR: Adjusted Odds Ratio, NS: Not Significant.

In terms of alcohol use, the results show that Vietnamese rural elderly who were male, 60-79 years old, married and having a caregiver reported higher percentage of drinking. However, after adjusting for potential confounding variables, only two variables (gender and age) were identified as significantly associated with the outcome of drinking among the elderly. Nevertheless, this model showed not a good fit to the data (p-value of the model coefficients <0.05; p-value of Hosmer and Lemeshow test $>0.05$ ). A similar pattern occurs with smoking behavior, which the subjects who were male, 60-79 years old, married and having a caregiver were likely to smoke than the others. Multivariate logistic regression analysis determined that gender, age, social participation, social cohesion and social support were the important variables which had significant association to smoking and this model was a good fit to the data with $\mathrm{R} 2=29.44 \%$, p-value of the model coefficients $<0.05$ and of Hosmer and Lemeshow test $>0.05$. Table 3 also reveals that the older people, who were female, were aged 80 years and older, single/divorce/widow and having a caregiver were likely to have a higher proportion of physical inactivity. Besides, associations were observed between physical inactivity and gender, marital status, having a caregiver, social participation, social cohesion and social support ( $\mathrm{p}$-value of the model coefficient $<0.05$, $\mathrm{p}$-value of Hosmer and Lemeshow test $>0.05$ and R2 $=15.25 \%$ ).

\section{Discussion}

The purpose of this study was to identify the magnitude and some associated factors of the three risky behaviors (drinking, smoking and physical inactivity) among the elderly in rural Vietnam. In our study, the prevalence of drinking alcohol of the subject was $25.17 \%$. This percentage was similar to the previous studies in southern provinces of Vietnam, Thailand, China $[12,15,20]$, but lower than that of some studies conducted in Western countries $[9,10,56]$. In terms of cigarette use, there were $22.17 \%$ of the older people reported as current smokers. This number was quite similar compared to the prevalence of the Vietnamese general population (23.8\%) [57] but higher than that of some other large-sample studies [28-30]. This high proportion of tobacco use can expose rural Vietnamese seniors at greater risks of smoking-related problems, as they are susceptible to health problems related to smoking. Notably the most prevalent risky behavior among seniors in our study was physical inactivity with $60 \%$ of the respondents who did not engage in any exercise activities at their age during the previous month. The result is in line with that of a systematic review of physical activity among older people with fiftythree included papers [58]. This high prevalence of physical inactivity may be explained in part due to the fact that the rural elderly still have to do their agriculture work so they spend their limited time to take a rest and not join any kinds of sports. The proportion of seniors who reported having both drinking and physical inactivity was $12.17 \%$, having both smoking and physical inactivity $11.17 \%$. This result was lower than that of the study conducted among older adults in Brazil with $64.6 \%$ and $62.3 \%$, respectively [41]. In particular, there were still $8 \%$ of the subjects who still had all three risky behaviors described above which may threaten their old age with a wide range of health problems. These high-risk subjects are urgently needed to be involved in some prevention programs to change their habits. Due to this high prevalence of risky behaviors, it is essential to find out the factors associated with each of these behaviors in this vulnerable population.

According to some research, drinking is a learned behavior that falls within the social norms of a cultural group $[59,60]$. This statement is pretty true in the context of Vietnam, where informal social drinking (nhậu) is the common way to create social networks, form business relationships and get respect from other people [61,62]. Using regression logistic analysis, our study indicated that gender and age were important predictors of alcohol use among the elderly in rural Vietnam $(\mathrm{p}<0.05)$. Specifically, the elderly who were male and younger were more likely to smoke than others. This is an expected finding and in concert with other studies which found striking gender differences in alcohol consumption between elderly men and women. Thus, the risks for health problems are several times higher among men than women [10,63]. Additionally, the younger elderly (60-79 years old) had significantly higher proportion of alcohol use than those aged more than 80. Many other studies also reported a similar reduction in alcohol use with growing age among the older population $[9,10,64]$. Alcohol consumption generally declines with age because of their changes in life circumstances and their awareness of increasing ill health. The relationship between drinking and social cohesion was reported by some previous studies [16,17]. However, our study did not show such a correlation. Besides, other predictors such as marital status, social support and social participation were not associated with drinking behaviors among the subjects. However, this regression logistic model is not a good fit to data with p-value of Hosmer and Lemeshow test $<0.05$. This can be explained that there were some other drinking-predicted factors among the elderly that we could not include in this study. Therefore, further research should be implemented to find out significant predictors to provide appropriate programs to reduce alcohol-related problems among the vulnerable elderly.

In rural Vietnam, the seniors usually smoke tobacco, especially bamboo pipe tobacco, to relax and socialize with others when they have free time. In terms of tobacco use, gender, age, social participation, social cohesion and social support had been shown to be significantly associated with smoking among the seniors $(\mathrm{p}<0.05)$. This is not surprising since men have a smoking prevalence many times higher than women, especially in Vietnamese culture which considers smoking as an antisocial behavior of women. Yi Wen and coworkers reported a similar observation with regard to Asian population [65]. Furthermore, higher age decreased the likelihood of smoking among the elderly with the significant odds. This result was in agreement with findings reported in studies involving other populations of seniors in the world $[29,66]$, due to the similar reason with drinking pattern that older seniors tend to reduce smoking to protect their susceptible health. In the current study, greater social cohesion was significantly correlated with an increased probability of smoking. In contrast, social participation may be a protective factor against smoking, which was a 
similar outcome with the study among Canadian elderly [67]. This might be partly because seniors who spend more time in social groups will have better knowledge about damaging effects of smoking through communication; therefore, they tend to quit or not use tobacco for a healthy lifestyle. The result according to social support showed a similar pattern. The elderly who have higher social support showed the significantly lower percentage of smoking. This could be explained by assuming that with support and encourage from surrounded people, the elderly have greater motivations for tobacco cessation and trying other healthy activities to improve their old age. Some other studies indicated that social cohesion was related with smoking cessation on adults group $[68,69]$; however, the present study showed the reversed effect among the elderly, perhaps because of the weak connection between the seniors and their neighborhood cohesion [70-72].

Doing exercises has proved to bring a great number of important quality-of-life and health benefits to older adults [37]. However, the proportion of seniors without regular physical activity was quite high in many populations [40,41,73] and can be explained by some associated factors. In our study, it was found that gender, marital status, having a caregiver, social participation, social cohesion and social support were important determinants of physical inactivity among the elderly $(\mathrm{p}<0.05)$. Precisely, females were more active in exercises compared to male seniors; however, this finding contrasted with the results of other studies which showed that physical activity was more likely among men $[41,58,73]$. Additionally, elderly who were single, divorced or widow were at greater risk of physical inactivity compared to married people. According to the multiple logistic regression model, social participation was a significant predictor which may encourage taking exercise among older adults. This is understandable because older people who involve in more social groups are likely to participate in social activities including exercises. This finding resembles some prior studies in both developing and developed countries $[41,73,74]$ and these similarities highlighted the importance of social involvement to participate in physical activities among the elderly. Some other studies indicated that social cohesion is associated with the well-being of older people [75,76]. In the present study, individuals who had greater social cohesion also had a significant higher likelihood of physical activity. However, social support was a negative predictor of doing exercises among the seniors. This can be understandable because this surveyed support was generally emotional or material support, which makes the elderly, enjoy their life and do not have to do hard activity, but not specifically support for doing exercise, which encourages seniors to participate in any kind of physical activity.

This study may have several limitations. First, in this study as we only assessed the risky behaviors through yes/no questions, the levels of smoking, drinking and physical inactivity were not classified, which does not allow to differentiate between minimal, moderate or excessive practice. Former studies in this field have indicated that - at least with regard to alcohol consumption - the dose seems to make the poison [77]. As this is a cross-sectional design, we would not able to assess the chronological development of risky behaviours with increasing age. Therefore, it might be limited when using the data to inform withinperson behaviour intervention. Accordingly, it is recommended that future research take into account a longitudinal design to allow for temporal causality and better design of interventions. Additionally, because of the limited research resource, physical inactivity was assessed through one variable "doing exercise or not" rather a multiitem scale. As was described above, one possible explanation for the low physical activity was that rural elderly still have to do their agriculture work. However, the respondents' leisure time activities or work-related duties were not explicitly assessed in the present study. Besides, the chosen period "last month" to assess one's own drinking and smoking behavior and the individual physical activity is likely to have an impact on the results. In this study, a time frame of "last month" was utilized to prevent recall loss; however, the results may be affected to some degree by some temporary factors such as acute physical pain or temporal accident. Further, although the multivariable models of predictors for risky behaviors were all of fit ( $p$-value of model cofficients $<0.05$ and $p$-value of Hosmer and Lemeshow indicators $>0.05$ ), the variance explained by independent variables in some models was not high, such as the model of predictors of physical inactivity $(\mathrm{R} 2=15.25 \%)$. This suggests that there possibly be other variables not included in the model, which would cause unobserved bias. For example, psychological variables such as self-efficacy have shown to be highly relevant in explaining health-related behavior in the elderly population [78]. Therefore, additional factors should be identified in future research to better explain the health-risk behaviors in Vietnamese rural older people. Besides, as this was a cross sectional study, we cannot ascribe causality to the associated factors in the results. Further prospective research should be carried out to strengthen and expand the results of this study.

\section{Conclusions and Implications}

This study was carried out in 600 older people in a rural province of Viet Nam. The results showed that the elderly in rural settings of Vietnam are engaged in multiple unhealthy behaviors, such as smoking, drinking, and physical inactivity. Factors associated with these unhealthy behaviors among the seniors are largely demographic and individual social factors. In particular, being male and 60-70 years old were associated with drinking in older people. Individuals, who were male, 60-79 years old, reported high social cohesion, low social participation and low social support were likely to smoke more than the others. Besides, some variables including being female, being married, having a caregiver, low social participation, social cohesion and higher social support were negatively associated with exercises among the older people after being adjusted for potential confounders. However, some of these factors like social participation and social support might be related to or affected by broader factors such as lack of social policies that support this group.

Recognizing that unhealthy behaviors among seniors are predicted by demographic and individual social factors enables us to design public health programs such as behavioral change communication programs. Considering the current level of risky behaviors including smoking, drinking and physical inactivity among the seniors, especially in rural areas where people have limited access to appropriate health and social services, it is necessary to have programs to decrease these risky behaviors. In terms of smoking and drinking behaviors, public health programs should be designed to specifically target older men, especially in rural areas with those who have lower education background and a lower awareness about the consequences of alcohol and tobacco. Health education and communication programs are expected to raise public and elderly awareness about risky behaviors and benefits of practicing healthy behaviors. To reduce drinking and smoking, it is also important to find ways to increase social support for the seniors to facilitate their behavior change. Due to the results which showed that social participation was significantly associated with reduced prevalence of smoking and physical inactivity among the elderly, there should be establishment of social networks for 
the seniors such as more clubs or social groups with diversified activities, so they are more likely to have chance to participate in. It is quite possible for the seniors as they have more time during their retirement. As Vietnam has much in common with other developing countries in Southeast Asia, the results of this study can be applied to similar countries for improving health conditions of the vulnerable rural elderly.

\section{Authors' Contributions}

DLM conducted statistical analyses, wrote and revised the manuscript. NVH provided technical guidance to the design analysis, and technically revised the manuscript. HS revised the manuscript and edited the language. NVT revised the manuscript and edited the manuscripts following the journal's requirements. All authors contributed and approved the final version of submitted manuscript.

\section{Acknowledgements}

We are indebted to the local authority and commune clinic centres whose useful supports profoundly facilitated our research implementation. We also express our deep gratitude to all of the elderly in rural Thai Binh provinces who took part and provided precious data to successfully complete this study.

\section{References}

1. United Nations (2013) World Population Ageing 2013. New York.

2. UNFPA (2011) The aging population in Vietnam - Current status, prognosis, and possible policy responses. Viet Nam.

3. Dall TM, Gallo PD, Chakrabarti R, West T, Semilla AP, et al. (2013) An aging population and growing disease burden will require a large and specialized health care workforce by 2025. Health Affairs 32: 2013-2020.

4. WHO (2011) Global Health and Ageing.

5. WHO (2014) Global status report on alcohol and health 2014.

6. Onder G, Landi F, Della Vedova C, Atkinson H, Pedone C, et al. (2002) Moderate alcohol consumption and adverse drug reactions among older adults. Pharmacoepidemiol Drug Saf 11: 385-392.

7. International Center for Alcohol Policies (2011) Practice Guides for Alcohol Policy and Prevention Approaches.

8. Institute of Alcohol Studies (2010) Alcohol and the Elderly.

9. Geels LM, Vink JM, HDA van Beek J, Bartels M, Willemsen G, et al. (2013) Increase in alcohol consumption in women and elderly groups: evidence from an epidemiological study. BMC Public Health 13: 207.

10. Hallgren M, Högberg P, Andréasson S (2009) Alcohol Consumption Among Elderly European Union Citizens: Health effects, consumption trends and related issues.

11. Weyerer S, Schäufele M, Wiese B, Maier W, Tebarth F, et al. (2011) Current alcohol consumption and its relationship to incident dementia: results from a 3-year follow-up study among primary care attenders aged 75 years and older. Age Ageing 40: 456-463.

12. Siviroj P, Pelzter K, Morarit S (2013) Alcohol Use and Associated Factors among Older Adults in Northern Thailand. Journal of Human Ecology 42: 187-193.

13. O'Connell H, Chin AV, Cunningham C, Lawlor B (2003) Alcohol use disorders in elderly people-redefining an age-old problem in old age. British Medical Journal 327: 664-667.

14. Peltzer K, Phaswana-Mafuya N (2013) Problem drinking and associated factors in older adults in South Africa. Afr J Psychiatry 16: 104-109.

15. Zhang J, Wu L (2015) Cigarette Smoking and Alcohol Comsumption among Chinese Older Adults: Do Living Arrangements Matter? Int J Environ Res Public Health 3: 2411-2436.
16. National Institute on Alcohol Abuse and Alcoholism (2004) Older Adults and Alcohol Problems.

17. Public Health Agency of Ireland (2013) Alcohol, drugs and older people.

18. Ganry O, Joly JP, Queval MP, Dubreuil A (2000) Prevalence of alcohol problems among elderly patients in a university hospital. Addition 95: 107-113.

19. Thang NT, Anh LV (2013) The elderly-valuable volunteers for the local health sector while participating in an active aging intervention program in Tien Hai district, Thai Binh province, Vietnam.

20. Ha NT, Le NH, Vushnu K, Rachael M (2015) Multimorbidity and its social determinants among older people in southern provinces, Vietnam. Int J Equity in Health 14: 50.

21. Leone A, Landini L, Leone A (2010) What is tobacco smoke? Sociocultural dimensions of the association with cardiovascular risk. Curr Pharm Des 16: 2510-2517.

22. Cataldo JK (2003) Smoking and Aging: Clinical Implications Part 1: Health and Consequence. J Gerontol Nurs 29: 15-20.

23. Tait R, Hulse G, Waterreus A, Flicker L, Lautenschlager NT, et al. (2006) Effectiveness of a smoking cessation intervention in older adults. Addition 102: 148-155.

24. Anstey KJ, Von Sanden C, Salim AO, Kearney R (2007) Smoking as a Risk Factor for Dementia and Cognitive Decline: A Meta-Analysis of Prospective Studies. Am J Epidemiol 166: 367-378.

25. Donze J, Ruffieux C, Cornuz J (2007) Determinants of smoking and cessation in older women. Age and Aging 36: 53-57.

26. Gons RA, Van Norden AG, de Laat KF, van Oudheusden LJ, van Uden IW, et al. (2011) Cigarette smoking is associated with reduced microstructural integrity of cerebral white matter. Brain 134: 2116-2124.

27. Nicita-Mauro V, Balbo C, Mento A, Nicita-Mauro C, Maltese G, et al. (2008) Smoking, aging and the centenarians. Exp Gerontol 43: 95-100.

28. Marinho V, Laks J, Coutinho ES, Blay SL (2010) Tobacco use among the elderly: a systematic review and meta-analysis. Cad Saude Publica 26: 2213-2233.

29. Lugo A, La Vecchia C, Boccia S, Murisic B, Gallus S (2013) Patterns of Smoking Prevalence among the Elderly in europe. Int J Environ Res Public Health 10: 4418-4431.

30. Liu F, Woodrow J, Loucks-Atkinson A, Buehler S, West R, et al. (2013) Smoking and alcohol consumption patterns among elderly Canadians with mobility. BMC Res Notes 6: 218.

31. Heshmati H, Charkazi A, Asnaashari R, Koohsar F (2014) Prevalence pf Smoking and the Related Factors Among the Elderly in Kashmar, Iran. Health Scope 2: 4.

32. Bui TV, Blizzard L, Luong KN, Truong Nle V, Tran BQ, et al. (2015) Declining Prevalence of Tobacco Smoking in Vietnam. Nicotine Tob Res 17: 831-838.

33. Nguyen Ngoc Dung (2015) Association between graphic health warning and reduction in cigarette demand among adult smokers: a study in some provinces in Vietnam 2014: Hanoi Medical University.

34. Xuan TLT, Minh HV, Giang KB, Nga PTQ, Hai PT, et al. (2013) Prevalence of Waterpipe Tobacco Smoking Among Population Aged 15 Years or Older, Vietnam, 2010. Preventing Chronic Disease 10: 120100.

35. Nguyen LT, Rahman Z, Mark RE, Nguyen MH, Zabin LS (2012) Cigarette Smoking and Drinking Behavior of Migrant Adolescents and Young Adults in Hanoi, Vietnam 50: S61-S67.

36. Minh HV, Hai PT, Giang KB, Kinh NL (2010) Prevalence of and susceptibility to cigarette smoking among female students aged 13 to 15 years in Vietnam, 2007. Prev Chronic Dis 7: A11.

37. WHO (2010) Global recommendations on Physical activity for Health.

38. WHO (2009) Global health risks: mortality and burden of disease attributable to selected major risks.

39. Pernambuco CS, Rodrigues BM, Bezerra JCB, Carrielo A, de Oliveira Fernandes AD, et al. (2012) Quality of life, elderly and physical activity. Health 4: 88-93. 
40. Chen Y, While AE, Hicks A (2015) Physical activity among older poeple living alone in Shanghai, China. Health Education Journal 74: 156-167.

41. Souza AMR, Fillenbaum GG, Blay SL (2015) prevalence and Correlates of Physical Inactivity among Older Adults in Rio Grande do Sul, Brazil. Plos ONE 10: 2 .

42. Lim K, Taylor L (2005) Factors associated with physical activity among older people-a population-based study. Prev Med 40: 33-40.

43. Belza B, Walwick J, Shiu-Thornton S, Schwartz S, Taylor M, et al. (2004) Older Adult Perspective on Physical Activity and Exercise: Voices from Multiple Cultures. Prev Chronic Dis 1: A09.

44. Bird S, Radermacher H, FeldmanS, Sims J, Kurowski W, et al. (2009) Factors influencing the physical activity levels of older people from culturally-diverse communities: an Australian experience. Aging Society 29: 1275-1294.

45. Thorpe JM, Thorpe CT, Kennelty KA, Pandhi N (2011) Patterns of perceived barriers to medical care in older adults: a latent class analysis. BMC Health Serv Res 11: 181.

46. Buckwalter KC, Davis LL (2008) Elder caregiving in rural communities. Rural Caregiving in the United States, pp: 33-46.

47. Goins RT, Williams KA, Carter MW, Spencer M, Solovieva T (2005) Perceived Barriers to Health Care Access Among Rural Older Adults: A Qualitative Study. J Rural Health 21: 206-213.

48. Mattson J (2010) Transportation, Distance and Health Care Utilization for Older Adults in Rural and Small Urban Areas.

49. Hopley M, Horsburgh M, Peri K (2009) Barriers to accessing specialist care for older people with chronic obstructive pulmonary disease in rural New Zealand. J Prim Health Care1: 207-214.

50. International Federation on Aging (2008) Vietnam Summary Report: Looking after and increasing the role of ageing in Viet Nam.

51. Knodel J, Anh TS (2002) Viet Nam's older population: the view from the census. Asia-Pacific Population Journal 17: 5-22.

52. Long GT, Pfau WD (2007) The elderly population in Vietnam during economic transformation: an overview. Social issues under economic transformation and integration in Vietnam 1: 185-210.

53. Kien Xuong District People's Committee (2012) Report "The population, economic, cultural, and social situation of Kien Xuong District in 2012 and orientations for development in 2013 ".

54. Prime Minister (2009) Decision 491/QĐ-TTg "Promulgating the national criteria for new rural".

55. Vietnamese Assembly (2009) Elderly Law, number 39/2009/QH12 on 23th, November, 2019.

56. JoAnn E, Kirchner CZ, Marisue Cody, Coakley E, Chen H, et al. (2007) Alcohol Consumption Among Older Adults in Primary Care. J Gen Intern Med 22: 92-97.

57. Ministry of Health of Vietnam, Hanoi Medical University GSO, CDC, WHO (2010) Global Adult Tobacco Survey (GATS) Viet Nam.

58. Sun F, Norman IJ, While AE (2013) Physical activity in older people: a systematic review. BMC Public Health 13: 449.

59. McCrady BS (2004) To Have But One True Friend: Implications for Practice of Research on Alcohol Use Disorders and Social Networks. J Addictive Behaviors 18: 113-121.
60. Rundle-Thiele S (2009) Social gain: Is corporate social responsibility enough? . Australasian Marketing Journal. 17: 204-210.

61. Parker L (2010) Alcohol Consumption behaviours and attitudes in Vietnam: An exploratory analysis. International Nonprofit \& Social Marketing Conference, Brisbane, Australia.

62. Ngoc LB, Thieng NT, Huong LN (2012) The drink driving situation in Vietnam. Traffic Injury Prevention 13: 109-114.

63. Institute of Alcohol Studies (2013) Older people and alcohol: Factsheet.

64. Wagner GA, Lebrão LM, Duarte YAdO, ZAnettaa DMT (2014) Alcohol Use among Older Adults: SABE COhort Study, São Paulo, Brazil. Plos ONE 9: e85548.

65. Tsai YW, TSAI TI, Yang CL, Kuo KN (2008) Gender differences in smoking behaviors in an Asia population. Journal of Women's Health 17: 971-978.

66. Peixoto SV, Araújo Firmo JO, Lima-Costa MF (2005) Factors associated to smoking habit among older adults. Rev Saúde Pública 39: 745-753.

67. Heather Gilmour (2012) Social participation and the health and wellbeing of Canadian seniors

68. Reitzel LR, Kendzor DE, Castro Y, Cao Y, Businelle MS, et al. (2013) The relation between social cohesion and smoking cessation among Black smokers, and the potential role of psychosocial mediators. Ann Behav Med 45: 249-257.

69. Fleischer NL, Lozano P, Arillo Santillán E, Reynales Shigematsu LM, Thrasher JF (2015) The impact of neighbourhood violence and social cohesion on smoking behaviours among a cohort of smokers in Mexico. J Epidemiol Community Health. 69: 1083-1090.

70. Dijk HV, Cramm JM, Nieboer AP (2008) Social cohesion as perceived by communitydwelling older people: the role of individual and neighbourhood characteristics. Int J Ageing Later Life 8: 9-31.

71. McPherson M, Smith-Loving L, Brashears ME (2006) Social isolation in America: Changes in core discussion networks over two decades. Am Sociol Rev 71: 353-375.

72. Oh JH, Aging KS (2009) neighbourhood attachment and fear of crime: Testing reciprocal effects. J Comm Psychol 37: 21-40.

73. Persson A, While A (2011) Physical activity among older people and related factors. Health Education Journal 71: 144-153.

74. Adams KB, Leibbrandt S, Moon H (2011) A critical review of the literature on social and leisure activity and well-being in later life. Ageing Societ 31: 683-712.

75. Cramm JM, Nieboer AP (2013) Relationships between frailty, neighborhood security, social cohesion and sense of belonging among community-dwelling older people. Geriatr Gerontol Int 13: 759-763.

76. Cramm JM, Nieboer AP(2015) Social cohesion and belonging predict the well-being of community-dwelling older people. BMC Geriatrics 15: 30 .

77. O'Keefe JH, Bhatti SK, Bajwa A, DiNicolantonio JJ, LavieCJ (2014) Alcohol and cardiovascular health: The dose makes the poison...or the remedy. Mayo Clin Proc 89: 382-393.

78. Grembowski D, Patrick D, Diehr P, Durham M, Beresford S, et al. (1993) Self-efficacy and health behavior among older adults. J Health Soc Behav 34: 89-104. 